\title{
Orbital Varices Revealed by Contusive Trauma: About A Case
}

\author{
Asmae Siati*
}

Department of Ophtalmology, Hospital August the thwentieth 1953, Ibn Rochd university hospital, Casablanca, Morocco

DOI: $10.36347 /$ simcr.2020.v08i03.009

| Received: 05.02.2020 | Accepted: 12.02.2020 | Published: 10.03.2020

*Corresponding author: Asmae Siati

Abstract

The etiologies of a unilateral exophthalmos are multiple, rarely represented by an intra- or extra-conical vascular mass. Orbito-palpebralvaricose veins are rare (2\% of orbital masses) and represent a major cause of unilateral intermittent exophthalmitis, often with an inflammatory character. We report a case of palpebral varices in a 45-year-old adult who underwent left orbital-facial contusion and who presented 2 months later a left-palpebral mass increasing in volume, extended to the left external canthus, associated with left exophthalmia intermittent, evolving for 6 months. Ophthalmologic examination revealed a moderate left ptosis with a left exophthalmia, non-axile, non-pulsatile, without thrill, painless, without complication, without visual deficit. A vascular mass is suspected on CT and MRI reveals a left palpebral varix with temporal extension, confirmed by ANGIO-MRI. The latter also made it possible to eliminate differential diagnoses (tumor, arteriovenous fistula, etc.) and to look for a cerebral venous malformation, an encephalocele or an associated bone defect. Without complications, treatment with platelet antiaggregant at low preventive dose was undertaken before the indication of a surgical excision for aesthetic damage and extensiveness. In case of complication, sclerosis, or even excision of the varicose vein, would be possible with disappointing results (recurrence, hemorrhage).

Keywords: Exophthalmia; orbit; varices; imagery.

Copyright @ 2020: This is an open-access article distributed under the terms of the Creative Commons Attribution license which permits unrestricted use, distribution, and reproduction in any medium for non-commercial use (NonCommercial, or CC-BY-NC) provided the original author and source are credited.

\section{INTRODUCTION}

The unilateral exophthalmos is most often due to an intraorbital expansive process. Orbital varices considered to be vascular tumors, are due to proliferation and dilation of intraorbital venous elements $[1,2]$. They represent $2 \%$ of orbital expansive processes $[1,2]$. They are revealed by an intermittent exophthalmia aggravated by the effort or the proclive position. We report a case of orbital varix revealed after a trauma.

\section{CASE RePORT}

This is a 45-year-old man who suffered a minor left orbito-facial contusion during a fall from his height hitting a table edge. No immediate complication was detected with normal radiological assessment. He consulted 8 months later for an upper left palpebral tumefaction extending temporally, evolving for 6 months, initially intermittent to postural, but constant and rapidly increasing in volume for 1 month. He has no other rhinological history. The ophthalmological examination found a left ptosis, a left exophthalmia non axile, painless, non-pulsatile. The examinations of the orbital bone frame, the refraction, the anterior segment as well as the fundus are without abnormality. The rhinological examination, cavum and cranial pairs were normal. The orbital CT revealed a lobular polyolevised left temporal palpebral and temporal formation, without close contact with the intra-conical content, without calcification within it. MRI confirms the presence of subcutaneous left palpebral and temporal subcutaneous vascular formation, measuring $73 \mathrm{~mm}$ wide and $31 \mathrm{~mm}$ anteroposterior diameter, with multiple hypointense T1 and T2 hyposignal structures (Figures 1 and 2) enhanced after injection in connection with a vascular platoon (Figure 3).

Our therapeutic approach was to initiate a low dose platelet antiaggregant treatment to prevent thrombosis. Later after preparation, a surgical excision for aesthetic damage was proposed to the patient. The indication is mainly raised because of intra-orbital nonextension of the lesion. The angiographic sequence shows drainage at the level of the facial vein, without further damage (Figure 4). 


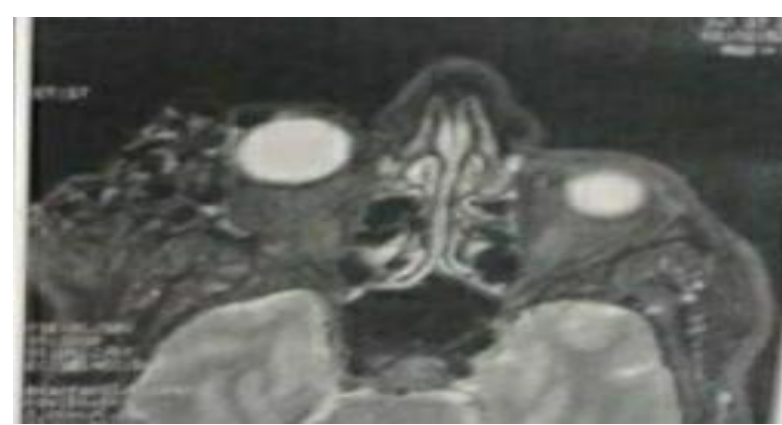

Fig-1: MRI in axial sections T1 vascularpalpebral and temporal hyposignal formation

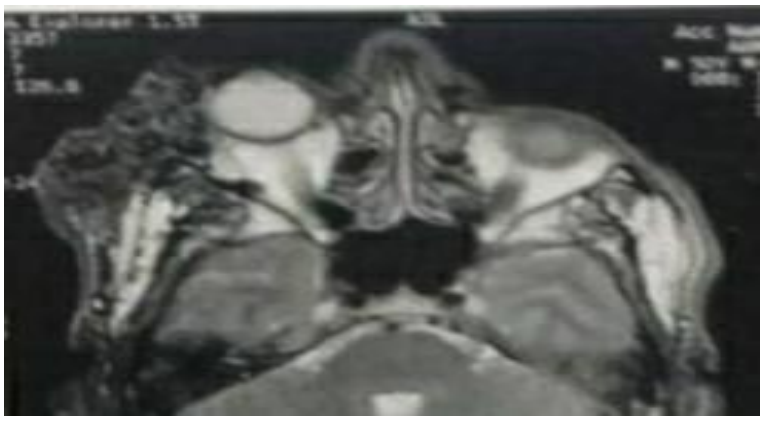

Fig-2: MRI in axial sections T2 vascularpalpebral and temporal hyposignal formation

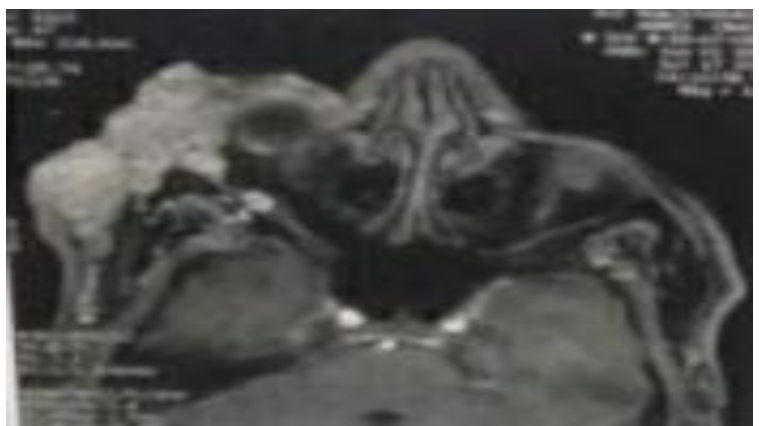

Fig-3: Axial MRI after gadolinium injection in FAT-SAT, showing intense enhancement of the lesion

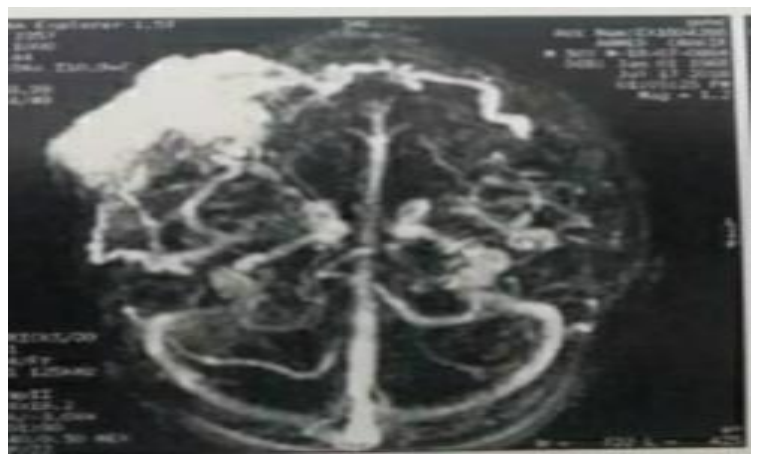

Fig-4: Angio-MRI sequence showing drainage of varicose vein in the facial vein

\section{DISCUSSION}

Tumors and vascular anomalies account for $10 \%$ of tumors in the orbit $[1,3]$. Of these tumors, hemangiomas, lymphangiomas, and orbital varices are the most common $[2,4]$. Orbital varices are congenital venous malformations, characterized by the proliferation of venous elements associated with the major dilation of one of the orbital veins. They can be congenital, idiopathic, post-traumatic, associated with a hemangioma or secondary to an arteriovenous fistula [1-4]. It evolves towards volume increase, thrombosis and endothelial hyperplasia. Clinically, orbital varicose veins are characterized by intermittent exophthalmitis occurring during exercise, proclive position, or Valsalva maneuver. This exophthalmosis non-pulsatile, with positional and sometimes painful variability [1, 3]. Imaging makes it possible to establish the diagnosis by comparing supine and procubitus images. It shows a formation that increases in volume by the operation of valsalva or in procubitus, and which enhances itself strongly with presence of evocative calcifications: phleboliths $[1,3]$. Treatment consists of surgical excision for symptomatic patients. Complications are an indication for surgery, such as sever eexophthalmia, orbital hemorrhage, compression of the optic nerve and posterior segment, or intolerable pain [5]. After surgery, recurrence is quite frequent, because of the posterior development of varicose veins near the optic nerve, and the important decrease of the volume of the lesion in decubitus and under general anesthesia. This makes complete excision difficult, and probable recurrences [1]. In the absence of complications careful monitoring should be preferred $[1,3]$.

\section{Conclusion}

Orbital varicose veins are generally revealed by an intermittent exophthalmitis, progressive evolution but can quickly evolve in case of triggering factor such as trauma, which is the case in our patient. In the absence of complications, therapeutic abstention is indicated with rigorous monitoring. Surgery is especially indicated for symptomatic patients with complication or aesthetic prejudice. It is noted a significant rate of recidivism.

\section{REFERENCES}

1. Cophignona J, d'Hermies F, Civit T. Tumeurs vasculairesde l'orbite. Neurochirurgie. 2010; 56:197-212.

2. Aydin A, Velioglu M, Ersanli D. Orbitalvarixpresentingwithenophthalmos. A case report. J. Fr Ophtalmol.2010;33:344.e1-5

3. Naggara O, Koskas P, Lafitte F, Heran F, Piekarski JD, Meder JF, Berges O. Vascular tumours and malformation of the orbit. Journal de radiologie. 2006 Jan;87(1):17-27.

4. Athanasiov PA, Prabhakaran VC, Selva D. Nontraumaticenophtalmos: areview. Acta Ophtalmol 2008;86:356-64.

5. Guigon-Souquet B, Grubain-Netter S, Macarez R,Giordano P, Bazin S. Varices intraorbitaire non compliquée :un problème thérapeutique difficile. $\mathrm{J}$ Fr Ophtalmol. 2002;25:840-2. 